American Journal of Agricultural and Biological Sciences 5 (3): 397-402, 2010

ISSN 1557-4989

(C) 2010 Science Publications

\title{
Fungal Culture Systems for Production of Antioxidant Phenolics Using Pecan Nut Shells as Sole Carbon Source
}

\author{
${ }^{1}$ Miguel A. Medina, ${ }^{1}$ Ruth E. Belmáres, ${ }^{2}$ Antonio Aguilera-Carbo, \\ Raúl Rodríguez-Herrera and ${ }^{1}$ Cristobal N. Aguilar \\ ${ }^{1}$ Department of Food Science and Technology, School of Chemistry, \\ Universidad Autonoma de Coahuila 25280, Saltillo, Coahuila, Mexico \\ ${ }^{2}$ Department of Food Science and Nutrition, Universidad Autonoma Agraria Antonio Narro, \\ 25350, Buenavista, Saltillo, Coahuila, Mexico
}

\begin{abstract}
Problem statement: Many agro-industrial wastes have little or none utilization, when these materials could be a very rich source of several value-added compounds, such as: the pecan nut shells, which contain Antioxidant Phenolic (AP) molecules like tannins. Approach: In this study, a bioprocess for the liberation of AP from Pecan Nut Shells (PNS) was described. A chemical characterization of raw material was evaluated to determine polyphenolic content of PNS, among other components. Several fungal culture systems were evaluated at $96 \mathrm{~h}$ fermentation processes and using PNS as sole carbon source. Solid (SSC) and Submerged (SmC) fermentations were carried out using three strains of Aspergillus niger. Culture medium was composed of a solid (10-50\%) and a liquid (90$50 \%$ ) part in order to complete the $100 \%$ of the medium. Results: A high concentration (19\%) of tannins was found in PNS on a dry basis, from which condensed tannins and their monomers were found as the main fraction (14\%) of tannins of PNS, also on a dry basis. In the strain selection step, Aspergillus niger GH1 showed better growth on pecan nut shells compared to A. niger PSH strain, therefore, A. niger GH1 was used for later experiments. The highest concentration of AP was obtained with SSC inoculated with spores of A. niger GH1 on the $40 \%$ of solids system and in $20 \%$ solids system where, among the other systems both presented $114 \mathrm{mg} \mathrm{TP} \mathrm{g}^{-1}$ of phenolics liberation at $24 \mathrm{~h}$ of fermentation. PNS is an important source of catechin, being this kind of compound which can be liberated in higher proportion compared to other phenolics compounds. Conclusion: This study demonstrated that fermenting PNS represent a good alternative to both use residues and obtaining AP.
\end{abstract}

Key words: Pecan nut shells, fungal culture systems, antioxidant phenolics, Solid and submerged fermentation, Aspergillus niger GH1 and PSH

\section{INTRODUCTION}

In North America, among the template weather zones of México and USA, the pecan nut tree grows producing nuts which have great demand in the food industry (Orzua et al., 2009). The main left-over of nut processing are the shells, which have in its composition many phenolics derived from tannins. There are four main groups of tannins, gallotannins, ellagitannins, condensed tannins and complex tannins (Khanbabaee and van Ree, 2001) According to other report, pecan nuts contain antioxidant phenolics and PNS contain higher amounts of these molecules, mostly condensed tannins (Villarreal-Lozoya et al., 2007). These compounds have many biological activities, such as antimicrobial, antimutagenic and antioxidant (AscacioValdes et al., 2010; Abdul Rahim et al., 2010). The recalcitrancy of these molecules are due to the strong protein binding activity, so microbes tend to inhibit their growth in presence of these compounds. However, there are few microorganisms able to degrade them. Filamentous fungi have been studied for biodegradation of tannins and liberation of phenolics (Aguilar et al., 2004; Mata-Gomez et al., 2009). According to these kind of experiments, filamentous fungi are capable of metabolize tannins and in a given moment, liberate tannin-monomers, which can be isolated. Solid state cultures are systems which have received praise due to the higher yields of compounds of interest than submerged cultures and also being a low cost

Corresponding Author: Cristobal N. Aguilar, Department of Food Science and Technology, School of Chemistry, Universidad Autonoma de Coahuila 25280, Saltillo, Coahuila, Mexico

Tel: +52(844)416 9213 Fax: +52(844)4390511 
technology (Alam et al., 2005). The objective of this work was to determine the capability of three Aspergillus niger strains to use pecan nut shells to liberate antioxidant phenolics under solid and submerged state culture and to detect and quantify the amounts of tannin derived AP resulting of these processes.

\section{MATERIALS AND METHODS}

Microorganism: For this study, three strains of Aspergillus niger (GH1, PSH and Aa-20), belonging to the DIA-UAdeC collection previously isolated, conserved and characterized by Cruz-Hernandez et al. (2005), were used.

Plant materials: The pecan nut shells were obtained from the Southern region of Coahuila State, Mexico, during the summer season of 2009 and transported to the Microbiology Laboratory of our institute. PNS were dried at sun light and stored in a black bag.

Content characterization: The chemical assays (protein, fat, ashes, crude fiber and nitrogen free extract) carried out were performed by previously established methods (Williams, 1984).

Strain selection for the bioprocesses: Once PNS were dehydrated, we proceeded to make a strain selection step. Three Aspergillus niger strains were used for this purpose, Aa-20 (control strain), PSH and GH1, fungal strains were growth on Petri dishes containing PNS and culture broth (Pontecorvo medium) in a 30:70 relation, in order to evaluate their adaptability on PNS. Once the system was ready, $50 \mu \mathrm{L}$ of spore suspension from the A. niger strains was placed in the center of the Petri dishes and mycelia growth was measured every $12 \mathrm{~h}$.

Solid and Submerged State Cultures (SSC and SmC): The culture processes were carried out using 5 different shell concentrations $(10,20,30,40$ and $50 \mathrm{~g})$ adjusted with Pontecorvo culture broth (90, 80, 70, 60 and $50 \mathrm{~mL}$, respectively). Culture broth composition was $\left(\mathrm{g} \mathrm{L}^{-1}\right) \mathrm{NaNO}_{3}(0.6), \mathrm{KH}_{2} \mathrm{PO}_{4}(0.152), \mathrm{KCl}(0.052)$, $\mathrm{MgSO}_{4} .7 \mathrm{H}_{2} \mathrm{O} \quad(0.052), \quad \mathrm{ZnSO}_{4}, \quad(0.0001)$. Other components of the broth were yeast extract at $0.05 \%$ and $1 \mathrm{~mL}$ of trace metals solution, with a composition as follow (mg. $\mathrm{L}^{-1}$ ) $\mathrm{Na}_{2} \mathrm{~B}_{4} \mathrm{O}_{7}$ (100), $\mathrm{Mn}_{2} \mathrm{Cl}_{4} \mathrm{H}_{2} \mathrm{O}$ (50), $\mathrm{NaMoO}_{4} \cdot 2 \mathrm{H}_{2} \mathrm{O}(50)$ and $\mathrm{CuSO}_{4} \cdot 2 \mathrm{H}_{2} \mathrm{O}(250)$, the broth $\mathrm{pH}$ was adjusted to 6.2 with $0.02 \mathrm{M} \mathrm{KOH}$. Pecan nut shells were the only carbon source in the systems and were inoculated with $2 \times 10^{7}$ spores of Aspergillus niger GH1 and incubated at $30^{\circ} \mathrm{C} 48 \mathrm{~h}^{-1}$. Samples were obtained every $12 \mathrm{~h}$ and stored at $-4^{\circ} \mathrm{C}$ in an ethanolwater mixture (1:1) until further analysis.
Total hydrolysable phenols (HT) and Gallic acid determination: The first determination was carried out using the Folin-Ciocalteu method (Makkar et al., 1995), the other one with the Sharma et al. (2000) method, both standard curves were prepared with gallic acid.

Condensed Tannins (CT): The method utilized was the reported by Schofield et al. (2001) using a catechin standard curve.

Ellagic acid determination: This analysis was carried out by HPLC using a gradient method and the conditions were: $1 \mathrm{~mL} \mathrm{m^{-1 }}$ flow, using a Triphasic pump (Varian model 230), autosampler (Varian 410), a photo diode array detector UV-Visible (Varian 330) and a Prodigy ODS $5 \mu$ (Phenomenex). The analysis conditions were as follow: A: Methanol, B: Acetonitrile and C: $0.3 \%$ Acetic acid at $0-5 \min 7 \%$ B-93\% C, 1213 min 60\% B-40\% C and 15-25 min 7\% B-93\% C.

Total Phenols (TP) during fungal culture: This parameter was quantified by the sum of the results of the Folin-Ciocalteu and the Schofield methods which were Gallic acid and catechin equivalents respectively.

\section{RESULTS}

Chemical characterization: The results which can stand out of the chemical characterization (Table 1) are the fiber content of 62.88 and $19.22 \%$ of tannin equivalents of both hydrolysable and condensed tannins, from which shells have $14.46 \%$ of condensed tannins and $5.43 \%$ of hydrolysable tannins. These compounds were molecules that matter the most to our work for the subsequent experiments.

Strain selection: Results of this study showed the capacity of three strains of using PNS as a sole carbon source. Aspergillus niger $\mathrm{GH} 1$ and Aa-20 could grow on PNS until $72 \mathrm{~h}$, where the experiment was stopped and $A$. niger PSH started and stopped its growth very quickly at 24 and $36 \mathrm{~h}$ respectively. The GH1 and Aa20 strains showed good adaptation capacity, so both strains were capable to degrade PNS components; many of those components are tannins. Narrowing the analysis on the $12 \mathrm{~h}$ of growth, it was observed that GH1 could grow even more rapidly than Aa-20, having a more stable growth (Fig. 1), even though, Aa-20 grew on the shells $12 \mathrm{~h}$ earlier than GH1. Past $12 \mathrm{~h}, \mathrm{GH} 1$ grows faster than the control strain, so GH1 must have a more developed tannin degrading enzymatic system than Aa-20. 


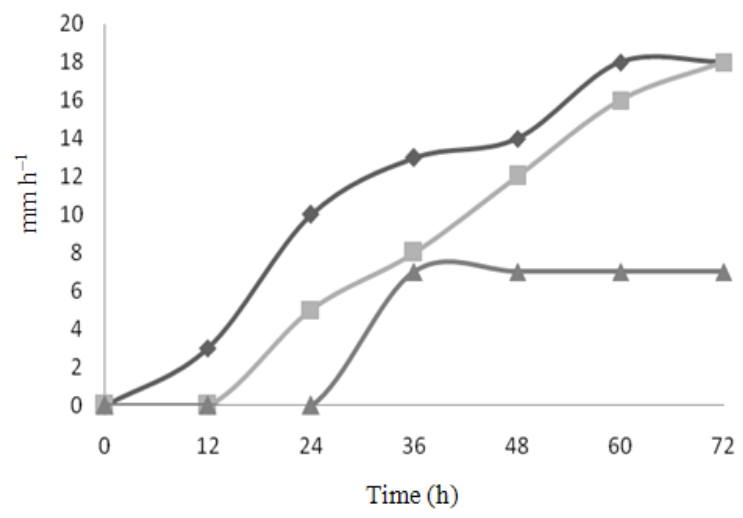

Fig. 1: Growth kinetic of Aspergillus niger GH1 ( $\square$ ), Aspergillus niger $\mathrm{PSH}(\Delta)$ and Aspergillus niger Aa-20 $(\diamond)$ strains on pecan nut shells

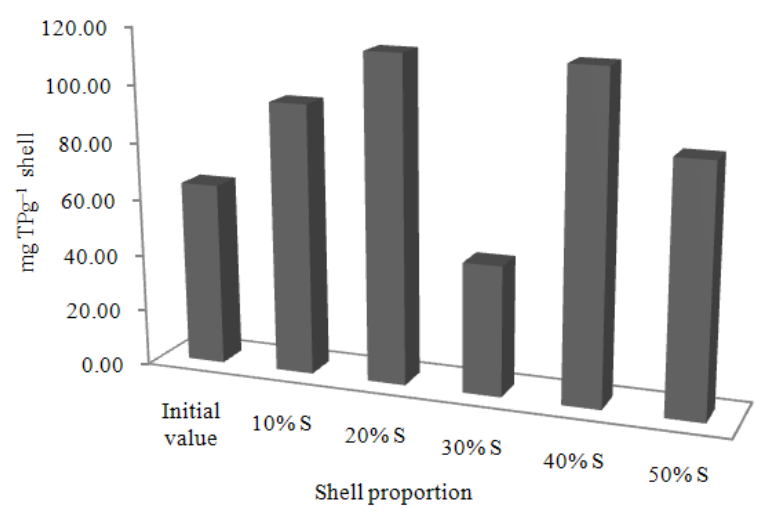

Fig. 2: Total phenolics liberated by Aspergillus niger GH1 from fermenting pecan nut shell at $12 \mathrm{~h}$ of culture

Table 1: Chemical content of the pecan nut shells

\begin{tabular}{lr}
\hline Parameters & Content $(\%)$ \\
\hline Dry matter & 91.90 \\
Humidity & 8.10 \\
Ashes & 2.28 \\
Protein & 4.29 \\
Fat & 1.36 \\
Fiber & 62.88 \\
Nitrogen free extracts & 29.19 \\
Total sugars & 2.83 \\
Reducing sugars & 0.03 \\
Hydrolyzable tannins (GAe) & 5.43 \\
Condensed tannins (Ce) & 14.46 \\
Total phenols & 19.12 \\
\hline
\end{tabular}

AP liberation: In almost every culture systems, the AP level was at its highest point at $12 \mathrm{~h}$. It was observed that in both 20 and $40 \%$ of PNS, the highest phenolic liberation was observed. The behavior of condensed tannins liberation (Fig. 3) showed high catechin concentration in the culture systems, while hydrolysable tannins had lower amounts.

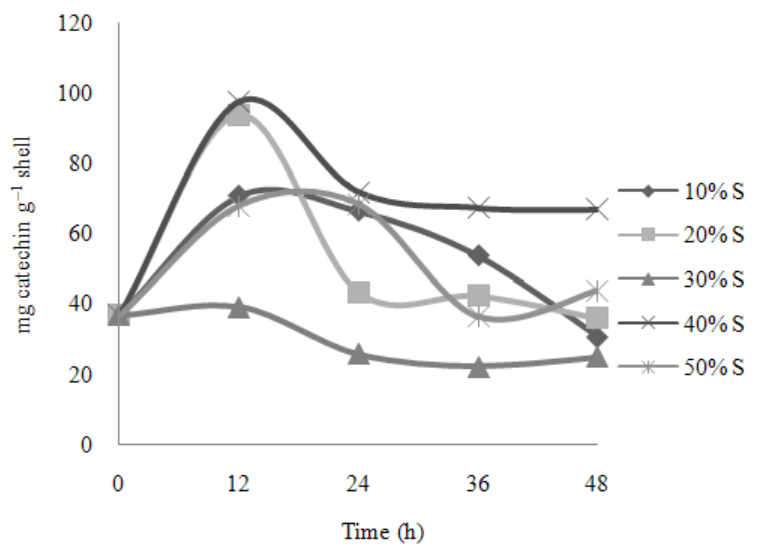

Fig. 3: Catechin equivalents liberated by Aspergillus niger $\mathrm{GH} 1$ al and from fermenting pecan nut shell

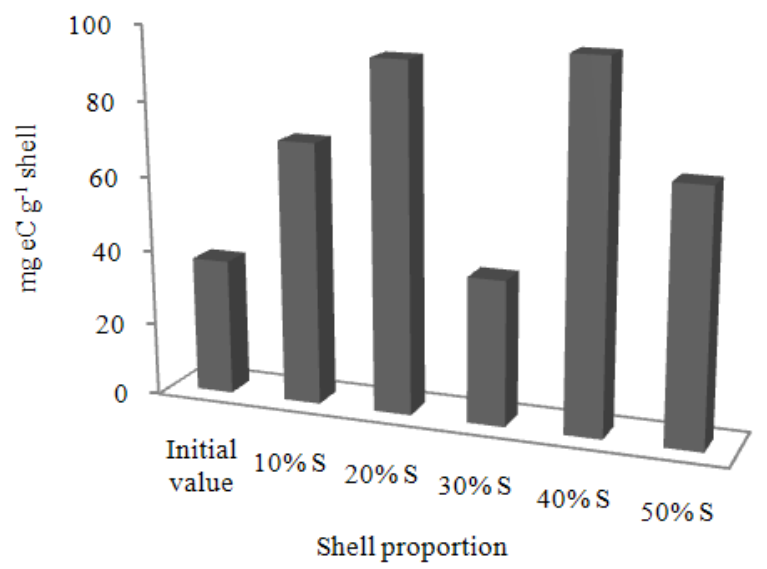

Fig. 4: Catechin equivalents at $12 \mathrm{~h}$ of culture

Having the sum of these compounds, in the Fig. 2 the TP pattern during the culture demonstrate that there is a phenolics liberation; consequence of the microbial hydrolysis of phenolics. In all systems, except 30\% shell, there's a steady liberation of TP where were registered in the 20 and $40 \%$ shells values up to 114.48 and $114.17 \mathrm{mg} \mathrm{TP} \mathrm{g}^{-1}$, followed by $10 \%$ shell with $95.31 \mathrm{mg} \mathrm{g}^{-1}$. All systems had an increase in both $\mathrm{CT}$ and HT at $12 \mathrm{~h}$ (Fig. 4). In this work, by the FolinCiocalteu assay, we could quantify up to $24.6 \mathrm{mg} \mathrm{g}^{-1}$ of HT in shells in the $10 \%$ shell system, in our process we obtained $114.48 \mathrm{mg} \mathrm{g}^{-1}$ of total phenolics by culture on the $20 \%$ PNS system. According to the chemical content, most of the phenolics present are CT so a major liberation of CT monomers could be expected. 


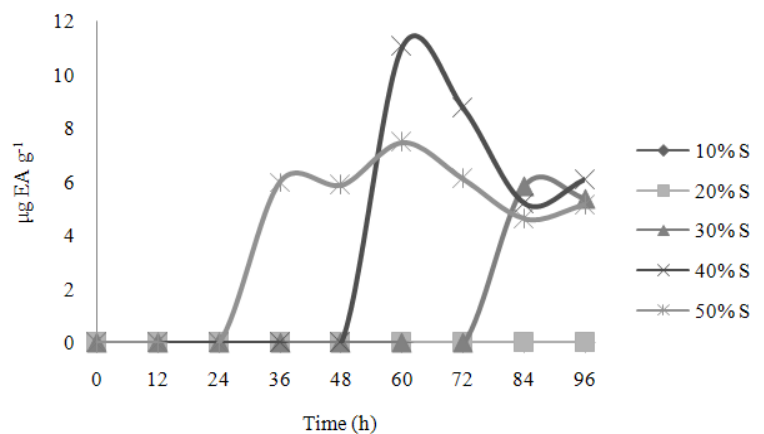

Fig. 5: Ellagic acid liberated by Aspergillus niger GH1 at from fermenting pecan nut shell

The gallic and ellagic acids contents were very small. Using the Sharma method, it was determined that only in the $10 \%$ shell system was possible to obtain gallic acid at $12 \mathrm{~h}$ of culture (data not shown) (Fig. 5). It was liberated $13.98 \mathrm{mg} \mathrm{g}^{-1}$ shell and in the other systems was obtained less than $1 \mathrm{mg} \mathrm{g}^{-1}$. In the $10 \%$ system, it was observed a gallic acid production, but, while the other systems did not showed positive results. The ellagic presence measured by HPLC analysis, showed that only the quantity obtained were in $\mu \mathrm{g} \mathrm{g}^{-1}$ shell and only in SSC, this behavior could be attributed to the insolubility of EA in water.

\section{DISCUSSION}

Results of this study showed that PNS can be used for fungal culture processes by Aspergillus niger strains, due to the fact that condensed tannins is the mayor component of the shells and the possibility of our strains being capable of degrade hydrolysable and condensed tannins (Aguilar et al., 2004). All used strains in the strain selection step, are reported as tannin degrading fungi (Cruz-Hernandez et al., 2005) and these same strains, were used in other similar growing experiments on tannin rich materials, where all of them were capable of growing on them (Orzua et al., 2009). Those experiments confirm the tannin degrading potential of these Aspergillus niger strains according to reports in literature (Bhat et al., 1998). As most of the tannins are of the condensed type, GH1 must have a more efficient mechanism for CT degradation and the enzymes required for that purpose, such as monooxygenases that cleave C-C bonds. Aspergillus niger GH1 must have also the necessary enzymes for the degradation of HT, which also are present in the PNS which is for example the tannin acyl hydrolase among others. The fungus is able to degrade tannins from the PNS after observing both growth and the results of the polyphenolic quantification. There are reports of condensed tannins degradation with fungal strains such as Penicillium expansum where taxifolin could be obtained as an intermediate in the condensed tannins degradation (Contreras-Dominguez et al., 2006), its liberation is due to monooxygenases produced by Aspergillus fumigatus that cleave the bonds between catechin units (Ramirez-Coronel et al., 2004), in this work no taxifolin was detected, but there is condensed tannins degradation. According to Bhat et al. (1998), some Aspergillus and Penicillium strains are capable of degrading both, hydrolysable and condensed tannins. Our results confirm the tannin degrading activity of Aspergillus niger GH1 over the tannins present in PNS, being this strain isolated from tannin rich materials, it was expected that this strain would be useful for AP liberation.

Aspergillus niger GH1 must synthesize the necessary enzymes for degradation those PNS components releasing phenolic monomers that form part of the polymeric structure of HT and CT; such enzymes are tannin-acyl hydrolase (Mingshu et al., 2006) and valonea tannase (Shi et al., 2005) or $\beta$ glucosidase (Vattem and Shetty, 2002) which have hydrolytic activity on ellagitannins and monooxygenases and dioxygenases responsible for the initial degradation steps of CT (ContrerasDominguez et al., 2006). Lewis and Starkey (1969) mentioned that some microorganisms isolated from soils are capable of degrading tannins from rich sources of these compounds, adding the fact that, there are studies where fungal strains can produce enzymes for CT degradation (Contreras-Dominguez et al., 2006) and others where Aspergillus niger GH1 was grown on a liquid culture with catechin (Aguilar et al., 2004), being this molecule the monomer unit of CT and tannic acid as sole carbon source (Mata-Gomez et al., 2009), so it can be said that this fungus can produce enzymes to degrade these kind of compounds and generate its metabolism energy even though presence of sugars, while limited, are not the only carbon source that could be used by the microorganism.

Some tannin rich materials have been used for AP liberation such as: Punica granatum (Robledo et al., 2008), coffee pulp (Pandey et al., 2000), Larrea tridentate (Mercado et al., 2007) and Fluorencia cernua (Ventura-Sobrevilla et al., 2008) most of the phenolics released in both cases were catechin equivalents, like in this case, most of the compounds liberation also were $\mathrm{CT}$ as catechin equivalents.

\section{CONCLUSION}

The pecan nut shells are an important source of antioxidant phenolics such as catechin and because it is 
a great agroindustrial residue, so an alternative biotechnological use has been developed exploiting the tannin degrading potential of Aspergillus niger GH1, which is capable of producing the enzymes responsible of the hydrolysis of CT and both, the filamentous fungi and the pecan nut shells have great possibilities for many other purposes that involves food and pharmaceutical industries.

\section{ACKNOWLEDGEMENT}

Researchers thank the financial support of the Mexican Council of Science and Technology (Fondo sectorial SAGARPA-CONACYT-2005).

\section{REFERENCES}

Aguilar, C.N., M. Cruz, R. Rodriguez, G. GutierrezSanchez and A. Ramirez-Coronel et al., 2004. Catechin degradation by several fungal strains isolated from Mexican desert. J. Microbiol. Biotechnol., 14: 1-4.

Alam, M.Z., N. Muhammad and M.E. Mahmat, 2005. Production of cellulase from oil palm biomass as substrate by solid state bioconversion. Am. J. Applied Sci., 2: 569-572. DOI: 10.3844/.2005.569.572

Ascacio-Valdes, J.A., A. Aguilera-Carbo, J.L. MartinezHernandez, R. Rodríguez-Herrera and C.N. Aguilar, 2010. Euphorbia antisyphilitica residues as a new source of ellagic acid. Chem. Papers, 64: 528-532. DOI: 10.2478/s11696-010-0034-6

Abdul Rahim, M.S.A., J. Salihou, M.M. Yussof, I. Abu Bakar and M.R.M. Damanik, 2010. Effect of temperature and time to the antioxidant activity in Plecranthus amboinicus Lour. Am. J. Applied Sci., 7: 1195-1199. DOI: 10.3844/.2010.1195.1199

Bhat, T.K., B. Singh, O.P. Sharma, 1998. Microbial degradation of tannins: A current perspective. Biodegradation, 9: 343-357. PMID: 10192896

Contreras-Dominguez, M., S. Guyot, N. Marnet, J.L. Petit, I. Perraud-Gaime and S. Roussos et al., 2006. Degradation of Procyanidins by Aspergillus fumigatus: Identification of a novel aromatic ring cleavage product. Biochimie, 88: 1899-1908. DOI: 10.1016/j.biochi.2006.07.011

Cruz-Hernandez, M., J.C. Contreras-Esquivel, F. Lara, R. Rodriguez and C.N. Aguilar, 2005. Isolation and evaluation of tannin-degrading fungal strains from Mexican desert. Z. Naturforsch, 60: 844-848. PMID: 16402543

Khanbabaee, K. and T. van Ree, 2001. Tannins: classification and definition. Nat. Prod. Rep., 18: 641-649. PMID: 11820762
Lewis, J.A. and R.L. Starkey, 1969. Decomposition of plant tannins by some soil microorganisms. Soil Sci., 107: 235-241.

Makkar, H., K. Becker, H. Abel and C. Szegletti, 1995. Degradation of condensed tannins by rumen microbes exposed to Quebracho Tannins (QT) in Rumen Simulation Technique (RUSITEC) and effects of QT on fermentative processes in the RUSITEC. J. Sci. Food Agric., 69: 495-500. DOI: 10.1002/jsfa.2740690414

Mata-Gomez, M., L.V. Rodriguez, E.L. Ramos, J. Renovato and M.A. Cruz-Hernandez et al., 2009. A novel tannase from the xerophilic fungus Aspergillus niger GH1. J. Microbiol. Biotechnol., 19: 987- 996. PMID: 19809257

Mercado, D., R. Belmares, A. Aguilera-Carbo, R. Rodriguez, N. Heredia and C.N. Aguilar, 2007. Toxicity and in vitro digestibility of creosote bush and tar bush fermented under fungal solid state culture conditions. Res. J. Biol. Sci., 2: 571-575.

Mingshu, L., Y. Kai, H. Quiang and J. Dongying, 2006. Biodegradation of gallotannins and ellagitannins. J. Basic Microbiol., 46: 68-84. DOI: 10.1002/jobm.200510600

Orzua, M.C., S.I. Mussatto, J.C. Contreras-Esquivel, R. Rodriguez and H.D.L. Garza et al., 2009. Exploitation of agro industrial wastes as immobilization carrier for solid-state fermentation. Ind. Crops Prod., 30: 24-27. DOI: 10.1016/j.indcrop.2009.02.001

Pandey, A., C.R. Soccol, P. Nigam, D. Brand and R. Mohan et al., 2000. Biotechnological potential of coffee pulp and coffee husk for bioprocesses. Biochem. Eng. J., 6: 153-162. DOI: 10.1016/S1369-703X(00)00084-X

Ramirez-Coronel, M.A., N. Marnet, V.S. Kumar Kolli, S. Guyot and S. Roussos et al., 2004. Characterization and estimation of proantocyanidins and other phenolics in coffee pulp (Coffea arabica) by thiolysis HPLC. J. Agric. Food Chem., 52: 1344-1349. DOI: 10.1021/jf035208t

Robledo, A., A. Aguilera-Carbo, R. Rodriguez, J.L. Martinez and Y. Garza et al., 2008. Ellagic acid production by Aspergillus niger in solid state culture of pomegranate residues. J. Ind. Microbiol. Biotechnol., 35: 507-513. DOI: 10.1007/s10295008-0309-X

Schofield, P., D.M. Mbugua and A.N. Pell, 2001. Analysis of condensed tannins: A review. Anim. Feed Sci. Technol., 91: 21-40. DOI: 10.1016/S0377-8401(01)00228-0 
Sharma, S., T.K. Bhat and R.K. Dawra, 2000. A Spectrophotometric Method Assay of Tannasse Using Rhodanine. Anal. Biochem., 279: 85-89. DOI: 10.1006/abio.1999.4405

Shi, B., Q. He, K. Yao, W. Huang and Q. Li, 2005. Production of ellagic acid from degradation of valonea tannins by Aspergillus niger and Candida utilis. J. Chem. Technol. Biotechnol., 80: 1154-1159. DOI: $10.1002 /$ jctb.1302

Vattem, D.A. and K. Shetty, 2002. Solid state production of phenolic antioxidants from cranberry pomace by Rhizopus oligosporus. Food Biotechnol., 16: 189-210. DOI: 10.1081/FBT120016667

Ventura-Sobrevilla, J., R. Belmares-Cerda, A. AguileraCarbo, J.C. Contreras and R. Rodriguez-Herrera et al., 2008. Fungal biodegradation of tannins from creosote bush (Larrea tridentata) and tar bush (Fluorencia cernua) for gallic and ellagic acid production. Food Technol. Biotechnol., 46: 211-215.
Villarreal-Lozoya, J.E., L. Lombardini and L. CisnerosZevallos, 2007. Phytochemical constituents and antioxidant capacity of different pecan [Carya illinoensis (Wangenh.) K. Koch] cultivars. Food Chem., 102: 1241-1249. DOI: 10.1016/j.foodchem.2006.07.024

Williams, S., 1984. Official Methods of Analysis of the Association of Official Analytical Chemists. 14th Edn., Association of Official Analytical Chemists, Washington DC., ISBN: 10: 0935584242, pp: 1141. 